\title{
Twist Periodic Solutions in the Relativistic Driven Harmonic Oscillator
}

\author{
Daniel Núñez and Andrés Rivera \\ Departamento de Ciencias Naturales y Matemáticas, Pontificia Universidad Javeriana, Seccional Cali, 26239 Cali, Colombia
}

Correspondence should be addressed to Andrés Rivera; amrivera@javerianacali.edu.co

Received 27 March 2016; Accepted 16 May 2016

Academic Editor: Svatoslav Staněk

Copyright (C) 2016 D. Núñez and A. Rivera. This is an open access article distributed under the Creative Commons Attribution License, which permits unrestricted use, distribution, and reproduction in any medium, provided the original work is properly cited.

We study the one-dimensional forced harmonic oscillator with relativistic effects. Under some conditions of the parameters, the existence of a unique stable periodic solution is proved which is of twist type. The results depend on a Twist Theorem for nonlinear Hill's equations which is established and proved here.

\section{Introduction and Main Results}

In this paper, we study the existence of a stable periodic solution (periodic response) in the one-dimensional forced harmonic oscillator with relativistic effects:

$$
\left(\frac{m x^{\prime}}{\sqrt{1-x^{\prime 2} / c^{2}}}\right)^{\prime}+k x=-F_{0} \cos \omega t,
$$

where $m>0$ is the mass at rest, $c>0$ is the speed of light in the vacuum, $k>0$ is the spring stiffness coefficient, and $F_{0}, \omega$ are the amplitude and frequency of the external force. Physically, we are assuming a basic principle of special relativity: the mass of a moving object is not constant but depends on its velocity. This equation can be derived from an appropriate Lagrangian or Hamiltonian formulation [1]. The Hamiltonian association of this system is

$$
H(x, y)=\sqrt{y^{2} c^{2}+m^{2} c^{4}}+\frac{1}{2} k x^{2}+x F_{0} \cos \omega t .
$$

The existence of chaotic behavior in the relativistic harmonic oscillator has been investigated numerically in [2]. More generally, the existence of chaotic dynamics of relativistic particles (relativistic chaos) has been reported in many different contexts [3-6].

From a more mathematical perspective, (1) can be seen as a singular $\phi$-Laplacian oscillator [7], then Landesman-Lazer condition holds, and there exists a periodic solution for all the values of the parameters. In other words, relativistic effects kill the classical linear resonance phenomenon.

Chu et al. have proven in [8] the stability of the equilibrium $x \equiv 0$ of the relativistic pendulum with variable length. We use a similar approach in order to prove the stability of a periodic solution for (1) obtained via lower and upper solutions in the reversed order (see Section 2).

On the other hand, in recent years some results about similar oscillators with relativistic effects, like the relativistic forced pendulum [9-11], have been published. There the authors have proven the existence and multiplicity of periodic solutions in the relativistic forced pendulum by variational and topological methods.

Our aim is to study the existence and stability of periodic solutions for the relativistic harmonic oscillator (1) with period $T=2 \pi / \omega$. We search $T$-periodic solutions so-called of twist type, meaning that its Floquet multipliers are not real and are not $n$ th-root of the unity for $n=1,2,3,4$, and its first Birkhoff's coefficient is different to zero (see Section 4 for definitions).

It is a well known fact that the appearance of $T$-periodic solution, of twist type, typically exhibits a KAM scenario around it, that is, existence of $n T$-periodic solutions for $n$ being arbitrary large in all neighborhood of it, some of which will be elliptic and the others will be hyperbolic. The hyperbolic solutions have generically transversal intersections between their associated stable and unstable manifolds $[12,13]$. 
The genericity is understood relative to certain topology constructed via jets of functions [13]. So, close enough to those intersections, certain invariant compact sets for the Poincaré transformation almost always (generically speaking) appear, called Smale's Horseshoe. A popular result in the theory of dynamical systems is that the dynamic on Smale's Horseshoe presents dense periodic orbits and sensitivity with respect to initial conditions and random itineraries (chaotic behavior).

According to this, the proof of the existence of periodic solutions of twist type is a first step in the comprehension of the chaotic behavior numerically evidenced in [2].

Theorem 1 is the main result of this paper.

Theorem 1. Assume that the parameters $m, k, \omega$, and $F_{0}$ satisfy the following conditions:

(H1) $k / m<\omega^{2} / 16$,

(H2) $F_{0}<(1 / 4) m c \omega$,

(H3) $\left((m c \omega)^{19}\left[(m c \omega)^{2}-16 F_{0}^{2}\right] / 120 \pi F_{0}^{2}\left(c^{2} m^{2} \omega^{2}+\right.\right.$ $\left.\left.4 F_{0}^{2}\right)^{19 / 2}\right) \sin \left(6 \pi \omega^{1 / 2} m c^{3 / 2} k^{1 / 2} /\left(c^{2} m^{2} \omega^{2}+4 F_{0}^{2}\right)^{3 / 4}\right)>$ 1 ,

and then the driven relativistic harmonic oscillator (1) has unique $2 \pi / \omega$-periodic solution which is of twist type and therefore Lyapunov stable.

Hypothesis (H3) may look rather weird; however, it gives some interesting corollaries in a direct way.

Corollary 2. With fixed $\omega, k, F_{0}$, and $c$ in (1), there exists $M_{0} \equiv$ $M_{0}\left(\omega, k, F_{0}, c\right)>0$ such that if $m>M_{0}$ then the conclusion of Theorem 1 holds.

Corollary 3. With fixed $m, k, F_{0}$, and $c$ in (1), there exists a critical frequency $\omega_{0} \equiv \omega_{0}\left(m, k, F_{0}, c\right)$ such that if $\omega>\omega_{0}$ then the conclusion of Theorem 1 holds.

Both corollaries follow easily by passing to the limit in the conditions of Theorem 1. Moreover, the critical values $M_{0}, w_{0}$ can be numerically computed. For instance, $M_{0}(1,1,1,1) \simeq$ 41.65497 and $\omega_{0}(1,1,1,1) \simeq 24.35658$.

The rest of the paper is organized as follows. Section 2 is devoted to analyze the existence of a periodic solution. As it is commented before, such existence is direct from the results of [7], although we present an alternative method which gives us some bounds for the solution. The key idea is the reduction of (1) to an equivalent Newtonian nonlinear oscillator. Then, the use of upper and lower solutions provides the existence as well as some bounds which are necessary in the sequel. Section 3 analyzes the uniqueness and linear stability of the periodic solution. Finally, in Section 4 a new Twist Theorem in the line of those presented in [14-16] is proved, which is applied in Section 5 in order to prove Theorem 1. Notice that it is not possible to apply directly Theorem 3.2 in [16] because the estimate (3.39) is not correct. The right estimate is established and proved in Lemma 8 , and this will be fundamental in order to establish the twist criteria (Theorem 7).

\section{Existence of Periodic Solutions}

As we mentioned in Introduction and Main Results, the existence of a periodic solution for (1) is a direct consequence of the results contained in [7]. We use here an alternative approach based on upper and lower solutions because it provides explicit bounds for the solution which will be crucial later.

For the Hamiltonian (2), we get Hamilton's equations

$$
\begin{aligned}
& x^{\prime}=\frac{c^{2} y}{\sqrt{y^{2} c^{2}+m^{2} c^{4}}}, \\
& y^{\prime}=-k x-F_{0} \cos \omega t .
\end{aligned}
$$

By deriving in the second equation, this system is equivalent to the second-order equation

$$
y^{\prime \prime}+f(y)=\omega F_{0} \sin \omega t
$$

with

$$
f(y)=\frac{k c^{2} y}{\sqrt{y^{2} c^{2}+m^{2} c^{4}}} .
$$

More precisely, notice that the associated first-order system for (4) with state variables $u=y$ and $v=y^{\prime}$ can be obtained from (3) by means of the following symplectic changes of variables with multiplier:

$$
\begin{aligned}
& u=y, \\
& v=-k x-F_{0} \cos (\omega t) .
\end{aligned}
$$

Thus, it is clear that the study of stability in both systems is equivalent, because the Poincaré mappings are conjugated. Therefore, the dynamics of a driven relativistic harmonic oscillator are equivalent to those of a driven nonrelativistic oscillator with the potential $V(y)=k \sqrt{y^{2} c^{2}+m^{2} c^{4}}$. This Newtonian equation has minimal period $T=2 \pi / \omega$ and we are interested in some key dynamical aspects like the existence of periodic solutions and its stability properties. The following result ensures us that (4) (as well as therefore (1)) has at least a $T$-periodic solution.

Proposition 4. Let one assume that $4 k<m \omega^{2}$. Then, (4) has a T-periodic solution $\varphi$ such that

$$
-\frac{F_{0}}{\omega}(1+\sin \omega t)<\varphi(t)<\frac{F_{0}}{\omega}(1-\sin \omega t)
$$

for all $t$.

Proof. It follows from the classical theory of upper and lower solutions [17, Theorem 4.1]. It is easy to verify that $\alpha(t)=$ $\left(F_{0} / \omega\right)(1-\sin \omega t)$ is a lower solution and $\beta(t)=-\left(F_{0} / \omega\right)(1+$ $\sin \omega t)$ is an upper solution such that $\beta(t)<\alpha(t)$ for all $t$. Moreover, note that $\left|f^{\prime}(s)\right| \leq k / m<(\pi / T)^{2}$, which is the condition for existence of a solution between the upper and the lower solution on the reversed order. 
Of course, the periodic solution $\varphi$ of (4) provides a periodic solution to the original equation (1) given by $x(t)=$ $-\left(\varphi^{\prime}(t)+F_{0} \cos \omega t\right) / k$.

\section{Uniqueness and Linear Stability}

In this section, we study the stability of the periodic solution found in the previous section in the linear sense. Let us fix a $T$-periodic solution $\varphi$ of (4), which always exists by Proposition 4 , and we translate it to the origin making the canonical changes of variables $x=y-\varphi(t)$. Thus, we lead to the equivalent equation

$$
x^{\prime \prime}+f(x+\varphi(t))-f(\varphi(t))=0 .
$$

Now the equilibrium $x \equiv 0$ is a solution. The linearization of $x=0$ is Hill's equation

$$
x^{\prime \prime}+a(t) x=0
$$

where

$$
a(t)=\frac{k c^{3} m^{2}}{\left(\varphi^{2}(t)+m^{2} c^{2}\right)^{3 / 2}} .
$$

By definition, $\varphi$ is said to be elliptic if the Floquet multipliers of Hill's equation (9) are complex conjugate numbers different from \pm 1 . An elliptic solution is in particular linearly stable.

The following bounds over $a(t)$ are easily deduced:

$$
\sigma_{1}^{2} \leq a(t) \leq \sigma_{2}^{2}
$$

with

$$
\begin{aligned}
\sigma_{1}^{2} & =\frac{k}{m}\left(1+\left(\frac{2 F_{0}}{c m \omega}\right)^{2}\right)^{-3 / 2}, \\
\sigma_{2}^{2} & =\frac{k}{m}
\end{aligned}
$$

Now we can formulate and prove the following result.

Proposition 5. Assume that

$$
4 k<m \omega^{2} \text {. }
$$

Then, (4) has a unique T-periodic solution $\varphi$ which is elliptic.

Proof of Proposition 5. From (13) and (11)-(12), we deduce that

$$
0<a(t)<\left(\frac{\pi}{T}\right)^{2}, \quad \forall t \in \mathbb{R} .
$$

Then, one application of the classical Lyapunov-Zukovskii stability criterion (see $[18,19]$ ) implies the ellipticity of (9).

For the uniqueness, suppose that $\psi(t)$ is another periodic solution. Then, $\phi-\psi$ is a T-periodic solution to Hill's equation:

$$
x^{\prime \prime}+h(t) x=0
$$

where

$$
h(t)= \begin{cases}\frac{f(\varphi(t))-f(\psi(t))}{\varphi(t)-\psi(t)} & \text { if } \varphi(t) \neq \psi(t) \\ f^{\prime}(\varphi(t)) & \text { otherwise. }\end{cases}
$$

By applying the mean value theorem and taking into account that $0<f^{\prime}<k / m$, we get

$$
0<h(t)<\frac{k}{m}<\left(\frac{\pi}{T}\right)^{2}
$$

for all $t$. Again, it follows that (15) is elliptic; therefore, the only $T$-periodic solution is the trivial one, so $\varphi=\psi$. This proves the uniqueness.

\section{Nonlinear Lyapunov's Stability}

The system under study is conservative, so the stability in the sense of Lyapunov can not be directly derived from the first approximation because of the possible synchronized influence of higher terms leading to resonance. After the works of Siegel and Moser [12], it is well known that the stability in the nonlinear sense depends generically on the third approximation of the periodic solution. So, we will focus on the third approximation for the reduced problem (4) around the periodic solution $\varphi$.

From the point of view of $\operatorname{KAM}$ theory $([12,20,21])$, the nonlinear terms of Taylor's expansion around a given periodic solution are taken into account to decide the kind of dynamic rising around such a solution. The basic idea consists in expressing the system in suitable geometrical coordinates as a perturbation of a canonical system which is integrable and therefore possesses invariant tori near to the periodic solution. These invariant tori are persistent under perturbations and produce jails or barriers for the flux trapping the orbits inside. As a by-product, it obtained the typical KAM scenario around the periodic solution (see $[12,22])$. The effective existence of a homoclinic transversal point for the Poincaré mapping $\mathscr{P}$ (which generates Smale's Horseshoe dynamics) is an interesting open question for this model. However, it is known that this property is generic for area preserving mappings $[13,22]$.

More recently, these ideas have taken a renewal interest starting from some Ortega's works $[14,23,24]$ that provide us with some stability criteria based on the third approximation. Some related references are $[15,16,25-29]$. We follow this approach and give a new stability criterion in line with those presented in [15, Theorem 2.2] and [16, Theorems 3.1 and 3.2].

Notice that the estimate (3.39) in [16] and, as a consequence, the condition (3.49) in Theorem 3.2 of that paper must be carefully reviewed. The right estimate is stated and proved in this section (Lemma 8). All these facts have motivated the rewriting of a clean criterion that works at least in the so-called first stability zone.

We consider the nonlinear Hill equation:

$$
x^{\prime \prime}+a(t) x+b(t) x^{2}+c(t) x^{3}+R(t, x)=0,
$$


where the functions $a, b, c: \mathbb{R} / T \mathbb{Z} \rightarrow \mathbb{R}$ are continuous, $b$ and $c$ are not both identically zero, and the remainder $R \in$ $C^{0, \infty}(\mathbb{R} / T \mathbb{Z}) \times(-\epsilon, \epsilon), \epsilon>0$, satisfies

$$
\frac{\partial^{k} R}{\partial x^{k}}(t, 0)=0, \quad k=0,1,2,3, \forall t \in \mathbb{R} .
$$

The solution $x \equiv 0$ is an equilibrium of (18).

The linearization of (18) at $x=0$ is Hill's equation:

$$
x^{\prime \prime}+a(t) x=0
$$

Let $M$ be the monodromy matrix of (20). The eigenvalues $\lambda_{1,2}$ of $M$ are called the Floquet multipliers of (20). The Floquet multipliers of (20) satisfy

$$
\lambda_{1} \lambda_{2}=1
$$

In a classical terminology, it is said that (20) (or $x=0$ ) is elliptic if $\lambda_{2}=\overline{\lambda_{1}} \in \mathbb{C}^{1} \backslash\{ \pm 1\}$, parabolic if $\lambda_{1,2}= \pm 1$, and hyperbolic if $\left|\lambda_{1,2}\right| \neq 1$, respectively. In the hyperbolic case, not only is the linear equation unstable but also $x=0$ like solution to (18).

Given $n \in \mathbb{N}$, we say that the equilibrium $x=0$ of (18) is $n$-resonant if it is elliptic and the Floquet multipliers satisfy $\lambda_{i}^{n}=1$. We say that $x=0$ is strongly resonant if it is $n$-resonant for $n=3$ or 4 .

The Poincaré mapping associated with (18) is defined near the origin by

$$
\mathscr{P}(x, y)=\varphi(T ; x, y)
$$

where $\varphi(t ; x, y)$ is the unique solution to (18) such that

$$
\begin{aligned}
\varphi(0 ; x, y) & =x, \\
\varphi^{\prime}(0 ; x, y) & =y .
\end{aligned}
$$

Note that $\mathscr{P}(0,0)=(0,0)$ and then the stability of $x \equiv 0$ (like the $T$-periodic solution to (18)) is equivalent to the stability of $(0,0)$ as fixed point of $\mathscr{P}$. The other elementary property of the Poincaré map states that $\mathscr{P}^{\prime}(0,0)$ is a monodromy matrix for (20), and then its eigenvalues are the Floquet multipliers of (20). If (20) is elliptic and not strongly resonant, by Birkhoff Normal Form Theorem there exists a canonical change of variables $z=\Phi(\xi)$ and $z=(x, y)$, such that $\mathscr{P}$ adopts in the new coordinates the following form:

$$
\mathscr{P}^{*}(\xi)=\left(\Phi^{-1} \circ \mathscr{P} \circ \Phi\right)(\xi)=R\left[\theta+\beta|\xi|^{2}\right](\xi)+O_{4},
$$

where $R[\delta]$ denotes the rotation of angle $\delta, \lambda=e^{ \pm i \theta}$ are the Floquet multipliers, and $\mathrm{O}_{4}$ indicates a term that is $O\left(|\xi|^{4}\right)$ when $\xi \rightarrow 0$. The coefficient $\beta$ is called the first twist coefficient and plays a central role in the stability. From the Twist Theorem it follows that if $\beta \neq 0$ then $(0,0)$ is stable (see $[12$, chapter 3]).

Definition 6. We say that the equilibrium $x \equiv 0$ of (18) is of twist type if it is elliptic and not strongly resonant and the associated first twist coefficient $\beta \neq 0$.
Notice that, according to this definition, all equilibrium of twist type is Lyapunov stable. Also, it is known, from the general theory, that an equilibrium of twist type exhibits $K A M$ dynamics around it as was mentioned in Introduction and Main Results.

The twist coefficient $\beta$ has an explicit formula and it is proportional to the integral quantity (see $[14,16]$ )

$$
\begin{gathered}
\beta^{*}=-\frac{3}{8} \int_{0}^{T} r(t)^{4} c(t) d t+\iint_{[0, T]^{2}} b(t) b(s) r^{3}(t) \\
\cdot r^{3}(s) \chi_{2}(|\phi(t)-\phi(s)|) d t d s,
\end{gathered}
$$

where $r(t)$ is the unique positive $T$-periodic solution to the Emarkov-Pinney equation ([16, Lemma 3.3]):

$$
y^{\prime \prime}+a(t) y=\frac{1}{y^{3}},
$$

the function $\phi$ is any primitive of $1 / r^{2}$, and the kernel function $\chi_{2}$ is defined by

$$
\begin{array}{r}
\chi_{2}(x)=\frac{2 \cos ^{3}(x-\theta / 2)+3 \cos \theta \cos (x-\theta / 2)}{8 \sin (3 \theta / 2)}, \\
x \in] 0, \theta] .
\end{array}
$$

The main result of this section is as follows.

Theorem 7. Assume that for (18) the third coefficient $c(t)<0$, $\forall t \in \mathbb{R}$. Choose $c_{*}>0$ and $b^{*}>0$ such that

(i) $-c(t)>c_{*}>0, \forall t \in \mathbb{R}$,

(ii) $|b(t)| \leq b^{*}, \forall t \in \mathbb{R}$.

Assume that the following conditions hold:

$$
\begin{aligned}
& 0<\sigma_{1}^{2} \leq a(t) \leq \sigma_{2}^{2}<\left(\frac{\pi}{2 T}\right)^{2}, \quad \forall t \in \mathbb{R}, \\
& \mathcal{c}_{*}>\frac{10 T \sigma_{2}{ }^{7}}{3 \sin \left((3 T / 2) \sigma_{1}\right) \sigma_{1}^{8}} b^{* 2} .
\end{aligned}
$$

Then, the equilibrium $x \equiv 0$ of (18) is of twist type.

Lemma 8. Assume that the condition (28) of Theorem 7 holds. Then,

$$
\frac{\sigma_{1}^{1 / 2}}{\sigma_{2}} \leq r(t) \leq \frac{\sigma_{2}^{1 / 2}}{\sigma_{1}}, \quad \forall t \in \mathbb{R} .
$$

Proof. The function $r(t)$ can be obtained by the following relation $([16$, Section 3.2]):

$$
r(t)=\alpha r^{*}\left(\frac{t-t_{0}}{\alpha^{2}}\right)
$$

for certain time scaling $\tau=\left(t-t_{0}\right) / \alpha^{2}$ which transforms Hill's equation $x^{\prime \prime}+a(t)=0$ into another one:

$$
x^{\prime \prime}+a^{*}(\tau) x=0, \quad a^{*}(\tau)=\alpha^{4} a\left(t_{0}+\alpha^{2} \tau\right)
$$


with new period $T^{*}=T / \alpha^{2}$ such that it is $R$-elliptic; that is, the associated monodromy matrix is a rigid rotation (see [14, Propositon 7]). The function $r^{*}$ is defined as $r^{*}=|\Psi|$, where $\Psi$ is the complex solution to (32) with initial conditions $\Psi(0)=1$ and $\Psi^{\prime}(0)=i$. Clearly, $a^{*}$ satisfies the same condition (28) of Theorem 7 with new constants $\sigma_{i}^{*}=\alpha^{2} \sigma_{i}$, $i=1,2$. So, $a^{*}$ holds the conditions of Lemmas 4.2 and 4.3(ii) of [15]. Combining these lemmas, we obtain

$$
\frac{1}{\alpha^{2} \sigma_{2}} \leq r^{*} \leq \frac{1}{\alpha^{2} \sigma_{1}}
$$

So, finally from the relation (31) we obtain the following uniform bounds on $r$ :

$$
\frac{1}{\alpha \sigma_{2}} \leq r \leq \frac{1}{\alpha \sigma_{1}} .
$$

From Lemma 4.3(ii), we know that $\sigma_{2}^{-1 / 2} \leq \alpha \leq \sigma_{1}^{-1 / 2}$, so finally we arrive from (34) to the required inequality.

Proof of Theorem 7. The proof of Theorem 7 follows basically the lines of [16]. For a function $f(t)$, let $f^{+}=\max \{f, 0\}$ and $f^{-}=\max \{-f, 0\}$ denote the positive and negative part of $f$. So, we can write $f=f^{+}-f^{-}$.

Condition (28) implies (see [15]) that $x \equiv 0$ is elliptic and not strongly resonant; therefore, the first twist coefficient $\beta$ is well defined. Remember that $\beta$ is proportional to $\beta^{*}$ given by (25). In order to prove Theorem 7, it is sufficient to show that $\beta^{*}>0$.

On the other hand, from (28) we also deduce that the rotation number $\rho$ associated with Hill's equation (20) satisfies

$$
\sigma_{1} \leq \rho \leq \sigma_{2}<\frac{\pi}{2 T}
$$

The characteristic exponent is by definition $\theta=T \rho$. Thus,

$$
\sigma_{1} T \leq \theta \leq \sigma_{2} T<\frac{\pi}{2}
$$

Let $r_{1}=\sigma_{1}^{1 / 2} / \sigma_{2}$ and $r_{2}=\sigma_{2}^{1 / 2} / \sigma_{1}$ denote, respectively, the lower and upper bound of $r(t)$ given by Lemma 8 . So, from (25) one deduces that

$$
\begin{aligned}
\beta^{*} & \geq \frac{3}{8} T r_{1}^{4} c_{*}+\iint_{[0, T]^{2}}\left(b^{+}(t)-b^{-}(t)\right) \\
\cdot & \left(b^{+}(s)-b^{-}(s)\right) r^{3}(t) r^{3}(s) \\
\cdot & \chi_{2}(|\phi(t)-\phi(s)|) d t d s \geq \frac{3}{8} \operatorname{Tr}_{1}^{4} c_{*} \\
- & \iint_{[0, T]^{2}} b^{+}(t) b^{-}(s) r^{3}(t) r^{3}(s) \\
\cdot & \chi_{2}(|\phi(t)-\phi(s)|) d t d s-\int_{[0, T]^{2}} b^{-}(t) b^{+}(s) \\
\cdot & r^{3}(t) r^{3}(s) \chi_{2}(|\phi(t)-\phi(s)|) d t d s,
\end{aligned}
$$

because the function $\chi_{2}$ is positive in $\left.(0, \theta] \subset\right] 0, \pi[$ (see (27) and (36)). On the other hand, an upper bound for $\chi_{2}$ was computed in [16]:

$$
\left.\left.\left|\chi_{2}(x)\right| \leq \frac{2+3 \cos \theta}{8 \sin (3 \theta / 2)}, \quad(x \in] 0, \theta\right], \theta \in\right] 0, \frac{\pi}{2}[) .
$$

Thus, using (36) and from the monotonocity of sin and cos in ] $0, \pi / 2[$, we get

$$
\left|\chi_{2}(x)\right| \leq \frac{2+3 \cos T \sigma_{1}}{8 \sin \left((3 T / 2) \sigma_{1}\right)}
$$

Going back to $\beta^{*}$, now we can deduce that

$$
\beta^{*} \geq \frac{3}{8} \operatorname{Tr}_{1}^{4} c_{*}-2 T^{2} r_{2}{ }^{6} b^{* 2} \frac{5}{8 \sin \left((3 T / 2) \sigma_{1}\right)}>0
$$

where the last inequality is a consequence of condition (29).

\section{Applications to the Relativistic Oscillator}

In Section 2, we prove the existence of a unique $2 \pi / \omega$ periodic elliptic solution $\varphi$ for the problem (4) under a suitable hypothesis over the parameters $k$ and $m$, more concretely, if $4 k<m \omega^{2}$. One more time, we emphasize that this problem is equivalent to the driven relativistic harmonic oscillator (1).

The third approximation for (4) around the periodic solution $\varphi$ is given by

$$
x^{\prime \prime}+a(t) x+b(t) x^{2}+c(t) x^{3}+\cdots=0
$$

where

$$
\begin{aligned}
& a(t)=\frac{k c^{3} m^{2}}{\left(\varphi^{2}(t)+m^{2} c^{2}\right)^{3 / 2}}, \\
& b(t)=\frac{-3 k m^{2} c^{3} \varphi(t)}{2\left(\varphi^{2}(t)+m^{2} c^{2}\right)^{5 / 2}}, \\
& c(t)=\frac{k m^{2} c^{3}\left(4 \varphi^{2}(t)-m^{2} c^{2}\right)}{2\left(\varphi^{2}(t)+m^{2} c^{2}\right)^{7 / 2}} .
\end{aligned}
$$

Proof of Theorem 1. From Proposition 5 and hyphothesis (H1), we have a unique $T$-periodic solution $\varphi$ which is elliptic and not strongly resonant and verifying the bound (7). In particular,

$$
|\varphi(t)|<\frac{2 F_{0}}{\omega}
$$

In order to prove that $\varphi$ is of twist type, we will apply Theorem 7 to the third approximation (41). By hyphothesis 
$(\mathrm{H} 2)$, we get $c(t)<0, \forall t \in \mathbb{R}$. Hence, the constants involved in Theorem 7 can be taken as

$$
\begin{aligned}
& \sigma_{1}^{2}=\frac{k}{m}\left(1+\left(\frac{2 F_{0}}{c m \omega}\right)^{2}\right)^{-3 / 2}, \\
& \sigma_{2}^{2}=\frac{k}{m}, \\
& b^{*}=\frac{3 k F_{0}}{\omega m^{3} c^{2}}, \\
& c_{*}=\frac{k m^{2} c^{3}\left(m^{2} c^{2}-16 F_{0}^{2} / \omega^{2}\right)}{2\left(m^{2} c^{2}+4 F_{0}^{2} / \omega^{2}\right)^{7 / 2}} .
\end{aligned}
$$

After several tedious computations, one can see that the inequalities (H3) of Theorem 1 and (29) of Theorem 7 are equivalent. The application of Theorem 7 finishes the proof.

\section{Competing Interests}

The authors declare that there is no conflict of interests regarding the publication of this paper.

\section{Acknowledgments}

This work is supported by Capital Semilla 2014-2015 project 00004025 Pontificia Universidad Javeriana, Seccional Cali, Cali, Colombia.

\section{References}

[1] H. Goldstein, Classical Mechanics, Addison-Wesley, Reading, Mass, USA, 2nd edition, 1980.

[2] J.-H. Kim and H.-W. Lee, "Relativistic chaos in the driven harmonic oscillator," Physical Review E, vol. 51, no. 2, pp. 15791581, 1995.

[3] M. Billardon, "Storage ring free-electron laser and chaos," Physical Review Letters, vol. 65, no. 6, pp. 713-716, 1990.

[4] C. Chen and R. C. Davidson, "Chaotic particle dynamics in freeelectron lasers," Physical Review A, vol. 43, no. 10, pp. 5541-5554, 1991.

[5] A. A. Chernikov, T. Tél, G. Vattay, and G. M. Zaslavsky, "Chaos in the relativistic generalization of the standard map," Physical Review A, vol. 40, no. 7, pp. 4072-4076, 1989.

[6] W. P. Leemans, C. Joshi, W. B. Mori, C. E. Clayton, and T. W. Johnston, "Nonlinear dynamics of driven relativistic electron plasma waves," Physical Review A, vol. 46, no. 8, pp. 5112-5122, 1992.

[7] C. Bereanu and J. Mawhin, "Existence and multiplicity results for some nonlinear problems with singular $\phi$-Laplacian," Journal of Differential Equations, vol. 243, no. 2, pp. 536-557, 2007.

[8] J. Chu, J. Lei, and M. Zhang, "The stability of the equilibrium of a nonlinear planar system and application to the relativistic oscillator," Journal of Differential Equations, vol. 247, no. 2, pp. 530-542, 2009.

[9] S. Maró, "Periodic solutions of a forced relativistic pendulum via twist dynamics," Topological Methods in Nonlinear Analysis, vol. 42, no. 1, pp. 51-75, 2013.
[10] H. Brezis and J. Mawhin, "Periodic solutions of the forced relativistic pendulum," Differential and Integral Equations, vol. 23, no. 9-10, pp. 801-810, 2010.

[11] C. Bereanu and P. J. Torres, "Existence of at least two periodic solutions of the forced relativistic pendulum," Proceedings of the American Mathematical Society, vol. 140, no. 8, pp. 2713-2719, 2012.

[12] C. L. Siegel and J. K. Moser, Lectures on Celestial Mechanics, Springer, New York, NY, USA, 1971.

[13] C. Genecand, "Transversal homoclinic orbits near elliptic fixed points of area-preserving diffeomorphisms of the plane," in Dynamics Reported: Expositions in Dynamical Systems, vol. 2, Springer, Berlin, Germany, 1993.

[14] R. Ortega, "Periodic solutions of a Newtonian equation: stability by the third approximation," Journal of Differential Equations, vol. 128, no. 2, pp. 491-518, 1996.

[15] D. Núñez, "The method of lower and upper solutions and the stability of periodic oscillations," Nonlinear Analysis: Theory, Methods \& Applications, vol. 51, no. 7, pp. 1207-1222, 2002.

[16] J. Lei, X. Li, P. Yan, and M. Zhang, "Twist character of the least amplitude periodic solution of the forced pendulum," SIAM Journal on Mathematical Analysis, vol. 35, no. 4, pp. 844-867, 2003.

[17] C. De Coster and P. Habets, "Upper and lower solutions in the theory of ODE boundary value problems: classical and recent results," in Non Linear Analysis and Boundary Value Problems for Ordinary Differential Equations, F. Zanolin, Ed., vol. 371 of CISM-ICMS Courses and Lectures, pp. 1-78, Springer, Vienna, Austria, 1996.

[18] W. Magnus and S. Winkler, Hill's Equation, Dover, New York, NY, USA, 1979.

[19] V. M. Starzinskii, "A survey of works on the conditions of stability of the trivial solution of a system of linear differential equations with periodic coefficients," American Mathematical Society Translations, vol. 1, pp. 189-237, 1955.

[20] V. Arnold, Méthodes Mathématiques de la Mécanique Classique, Mir, Moscow, Russia, 1976.

[21] J. Möser, On Invariant Curves of Area-Preserving Mappings of an Annulus, Nachrichten der Akademie der Wissenschaften in Göttingen. II Mathematisch-Physikalische Klasse II, Vandenhoeck \& Ruprecht, Göttingen, Germany, 1962.

[22] S. E. Newhouse, "Quasi-elliptic periodic points in conservative dynamical systems," American Journal of Mathematics, vol. 99, no. 5, pp. 1061-1087, 1977.

[23] R. Ortega, "The stability of the equilibrium of a nonlinear Hill's equation," SIAM Journal on Mathematical Analysis, vol. 25, no. 5, pp. 1393-1401, 1994.

[24] R. Ortega, "The twist coefficient of periodic solutions of a time-dependent Newton's equation," Journal of Dynamics and Differential Equations, vol. 4, no. 4, pp. 651-665, 1992.

[25] B. Liu, "The stability of the equilibrium of a conservative system," Journal of Mathematical Analysis and Applications, vol. 202, no. 1, pp. 133-149, 1996.

[26] D. Núñez and R. Ortega, "Parabolic fixed points and stability criteria for non-linear Hill's equation," Zeitschrift für Angewandte Mathematik und Physik ZAMP, vol. 51, no. 6, pp. 890911, 2000

[27] D. Núñez and P. J. Torres, "Periodic solutions of twist type of an earth satellite equation," Discrete and Continuous Dynamical Systems, vol. 7, no. 2, pp. 303-306, 2001. 
[28] D. Núñez and P. J. Torres, "Stable odd solutions of some periodic equations modeling satellite motion," Journal of Mathematical Analysis and Applications, vol. 279, no. 2, pp. 700-709, 2003.

[29] D. Núñez and P. J. Torres, "KAM dynamics and stabilization of a particle sliding over a periodically driven curve," Applied Mathematics Letters, vol. 20, no. 6, pp. 610-615, 2007. 


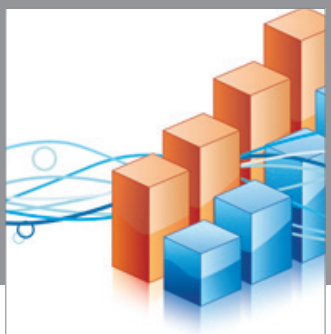

Advances in

Operations Research

vatem alat4

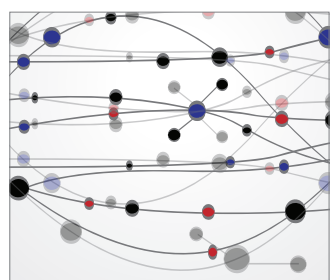

\section{The Scientific} World Journal
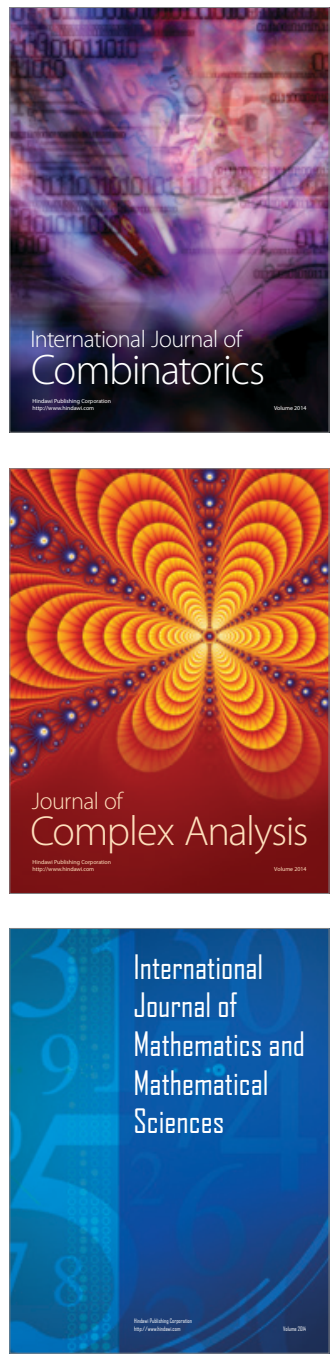
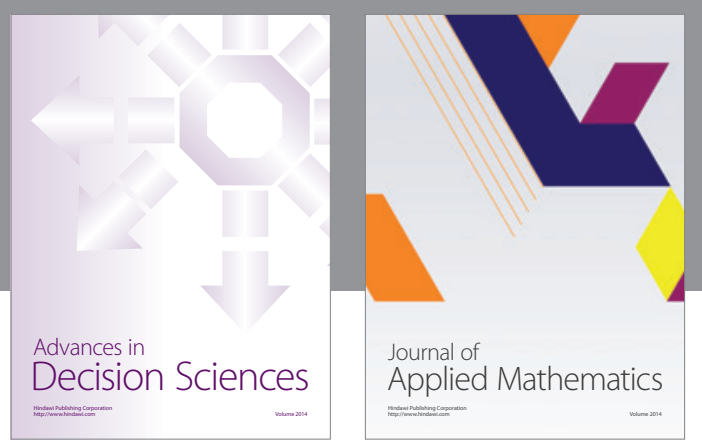

Algebra

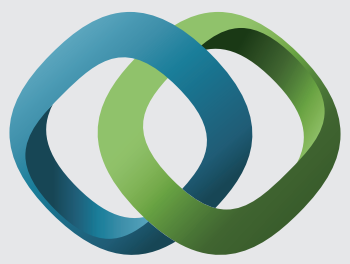

\section{Hindawi}

Submit your manuscripts at

http://www.hindawi.com
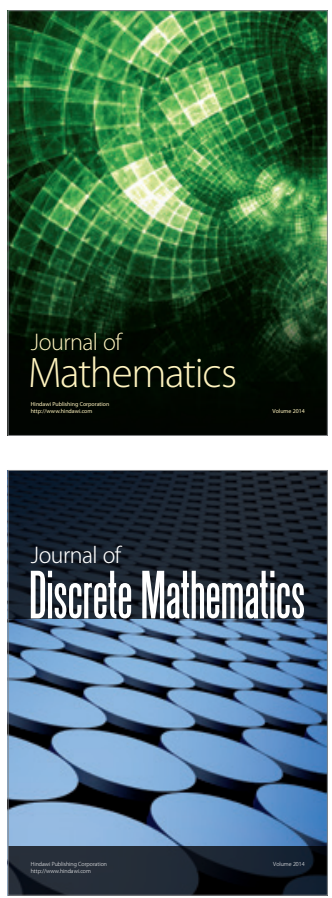

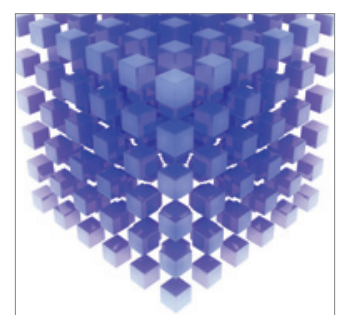

Mathematical Problems in Engineering
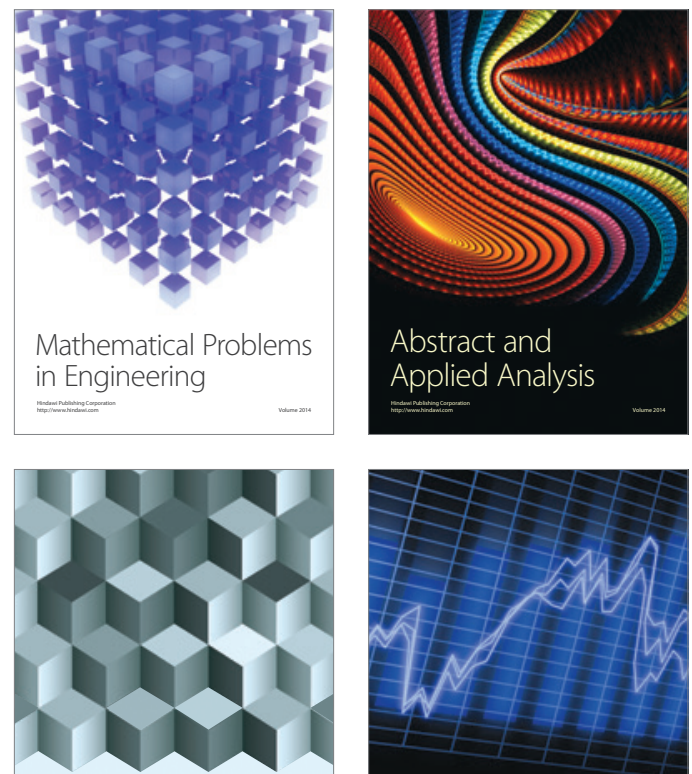

Journal of

Function Spaces

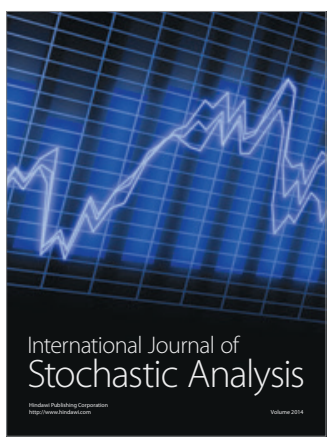

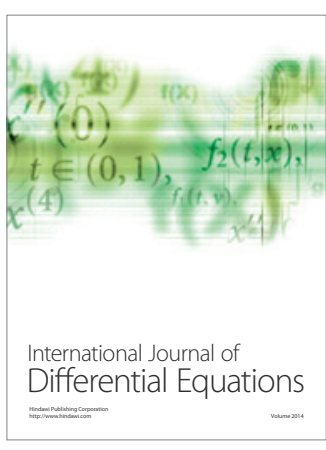
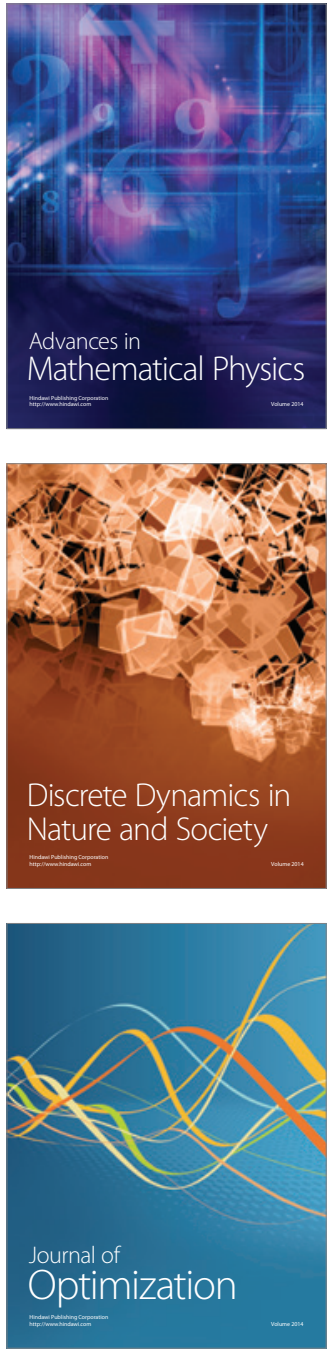